\title{
Effect of Decaffeinated Coffee Consumption on Markers of Atherogenesis and Carbohydrate Metabolism in Diet-Induced Dyslipidemia in Rats
}

\author{
Christopher Edet Ekpenyong $^{1 *}$ Daniel Emmanuel Otokpa ${ }^{2}$ \\ ${ }^{1}$ Department of Physiology (Cardio-renal Unit), Faculty of Basic Medical Sciences, University of Uyo, \\ Uyo, Nigeria \\ ${ }^{2}$ Department of Medicine (Renal Unit) University of Calabar Teaching Hospital, Calabar, Nigeria
}

Received Date: September 10, 2020; Accepted Date: September 21, 2020; Published Date: September 29, 2020;

"Corresponding author: Christopher Edet Ekpenyong, Department of Physiology, Faculty of Basic Medical Sciences, University of Uyo, Uyo, Nigeria. Email: chrisvon200@yahoo.com, chrisvon300@yahoo.com

\section{Abstract}

There are contradictory opinions about the effect of coffee on serum markers of atherogenesis in the literature. This study aimed to assess the effect of decaffeinated coffee consumption on serum lipid levels, atherogenic index of plasma (AIP), and markers of carbohydrate (CHO) metabolism, in diet-induced dyslipidemia in rats.

Twenty-five Wistar albino rats were divided into 5 groups ( $\mathrm{n}=5$ per group). Animals in group 1 served as a normal control group (NCTRL) and were given a normal diet and water only. Animals in group 2 received a hyperlipidemic diet. Animals in groups 3,4 , and 5 in addition to the hyperlipidemic diet were treated with low, medium, and high doses of decaffeinated coffee respectively for 28 days. At the end of the coffee treatment, and $8 \mathrm{~h}$ after feeding, all animals were sacrificed and blood obtained was analyzed for the biochemical indices of atherogenesis and $\mathrm{CHO}$ metabolism using standard methods.

There were significant $(\mathrm{P}<0.05)$ increases in serum levels of total cholesterol (TC), triglyceride cholesterol (TG-C), lowdensity lipoprotein cholesterol (LDL-C), very low-density lipoprotein cholesterol (VLDL-C), AIP, and markers of CHO metabolism (FBG, insulin, and Homeostatic Model Assessment of Insulin Resistance (HOMA-IR)), as well as a decrease in highdensity lipoprotein cholesterol (HDL-C) in the hyperlipidemic alone group. Treatment with different doses of decaffeinated coffee caused a reversal of these indices to near levels in the control group in a dose-dependent manner, especially in the medium-dose group.

Moderate consumption of decaffeinated coffee can ameliorate diet-induced dyslipidemia and improved markers of $\mathrm{CHO}$ metabolism.

Keywords: Atherogenesis; Coffee; Hyperlipidemic Diet; Serum Lipids

\section{Introduction}

Dyslipidemia is a metabolic disorder characterized by high low-density lipoprotein cholesterol (LDL-C), high total blood cholesterol (T-chol), low level of high-density lipoprotein cholesterol (HDL-C), and hypertriglyceridemia. It is one of the most important factors for the development of atherosclerosis that leads to coronary heart diseases (CHD). CHD is the most common cause of death among women [1], and in particular, high triglyceride (TG) to low HDL-C (TG/HDL-C) ratio is a powerful independent predictor of all-cause mortality and cardiovascular event. High TG-C and low HDL-C when cooccurring with elevated Apo-protein B and small dense LDL$\mathrm{C}$ particles are strongly predictive of metabolic syndrome and CVD morbidity in the general population [2].

The status for dyslipidemia varies from one individual to another and may be influenced by some non-modifiable and modifiable factors including age, ethnicity, genetics, and lifestyle factors such as diet. From the dietary perspective, a 
noteworthy link exists between diet and abnormal serum lipid levels. Evidence has been garnered indicating that diet high in saturated fatty acids and cholesterol, but low in fibers, plant foods, and unsaturated fatty acids drive abnormal serum lipid profile and atherosclerosis [3]. Conversely diets low in fat, but high in fruits, vegetables, plant foods is the key to lowering blood lipid and keeping dyslipidemia in control and manageable level.

Given the enormous side effects associated with the use of synthetic lipid-lowering drugs (fibrates and statins), there is a fervent search for dietary options to complement drug therapy. Nutritional modification could lead to a reduction in dose and duration of use of drugs and by extension reduction in adverse health effects of drug therapy.

Coffee is one such plant-derived food which although widely consumed and socially accepted all over the world has not been adequately evaluated for its role in dyslipidemia. Coffee is a brewed beverage prepared from the roasted seeds of several species of an evergreen shrub of the genus Coffee.

Coffee seed apart from the caffeine content has several bioactive constituents and consumption of coffee tea has been shown to provide some health benefits including a reduction in the risk of Alzheimer's disease, dementia [4] Parkinson's disease, heart disease, diabetes mellitus, cirrhosis, and gout.The present study aimed to evaluate the effect of coffee on serum uric acid (SUA), glucose and log TG/HDL-C and markers of insulin resistance (IR) in the animal model of dyslipidemia.

\section{Materials and Methods}

\section{Selection/treatment of experimental animals}

Twenty-five matured male Wistar albino rats weighing 170-233g were obtained from the animal house at the Faculty of Basic Medical Sciences, University of Uyo, Nigeria. The rats were kept in standard stainless steel cages for 14 days for acclimatization. Their body weight was monitored before and after the experiment.

They were kept on a $12 \mathrm{~h}$ light/dark cycle, at room temperature $\left(25-29^{\circ} \mathrm{C}\right)$, and with a relative humidity of $56 \% \pm$ $8 \%$. All animals were fed standard animal feed and water adlibitum. After the period of acclimatization, the rats were randomly divided into 5 groups ( $n=5$ per group). Group 1 served as normal control and was administered normal feed and water only. Groups 2, 3, 4, and 5 were fed a hyperlipidemic diet $(\mathrm{g} / 100 \mathrm{~g}$ of normal diet) consisting of egg yolk $35 \mathrm{~g}$, cholesterol $0.5 \mathrm{~g}$, fish oil, and sunflower oil $20 \mathrm{~g}[\mathbf{5}, \mathbf{6}]$.

After the determination of LD50 for coffee, animals in groups 3,4 , and 5 were concomitantly treated with low $(0.20 \mathrm{ml})$, medium $(0.40 \mathrm{ml})$, and high dose $(0.65 \mathrm{ml})$ respectively of diluted (decaffeinated coffee-Nestle Gold, UK) once daily (morning) by oral gavage for 28 days. Animals in group 2 served as hyperlipidemic diet (HLD) control.

\section{Biochemical Analyses}

At the end of coffee administration (i.e., 28 days after) and $8 \mathrm{~h}$ after feeding, all animals were anesthetized with chloroform, and blood was obtained by cardiac puncture into specimen bottles with no anti-coagulant. Serum lipid sub-fractions including HDL-C, TG-C, T-chol, and VLDL-C were determined using lipid profile automated measuring system (Lipid proTM), model ILM-001A. Info pia Co. Ltd. South Korea. LDL-C was calculated using the empirical relationship of Friedewald equation; [LDL=TC-HDL-C-TG/5(when TG<400mg/dl)] [7].

The SUA level and homeostatic model assessment of insulin resistance (HOMA-IR) were also determined using standard methods. Atherogenic index of plasma (AIP) defined as the ratio of $\log$ TG/HDL-C was determined, where TG represents serum triglyceride and HDL-C represents serum high-density lipoprotein cholesterol.

All research protocols were conducted at the University of Uyo in line with the Nigerian and International laws governing the acceptable use of laboratory animals.

\section{Statistical analysis}

Values are reported in the form of mean \pm standard deviation. Differences in the parameters between the groups were examined using Analysis of Variance (ANOVA) followed by the Duncan test to separate the means and determine which of the group is significantly different from the other. To enhance the computation of results, the Statistical Package for Social Sciences was used while graph pad prism 7.0 was used to produce the graphs.

\section{Results}

\section{Serum total cholesterol (T-chol)}

Serum T-chol increased significantly in the hyperlipidemic diet alone group compared with the control group. Hyperlipidemic diet plus low dose decaffeinated coffee caused a significant increase in serum T-chol compared with the control group, but when compared with the hyperlipidemic group, a null significant difference was observed.

Hyperlipidemic diet plus a medium dose of decaffeinated coffee caused a significant decrease in serum T-chol when compared with control and hyperlipidemic diet alone groups. Furthermore, hyperlipidemic diet plus high dose decaffeinated group was associated with a nonsignificant change in serum $\mathrm{T}$ chol when compared with the control group, but a significant 
decrease when compared with the hyperlipidemic diet alone group (Figure 1).

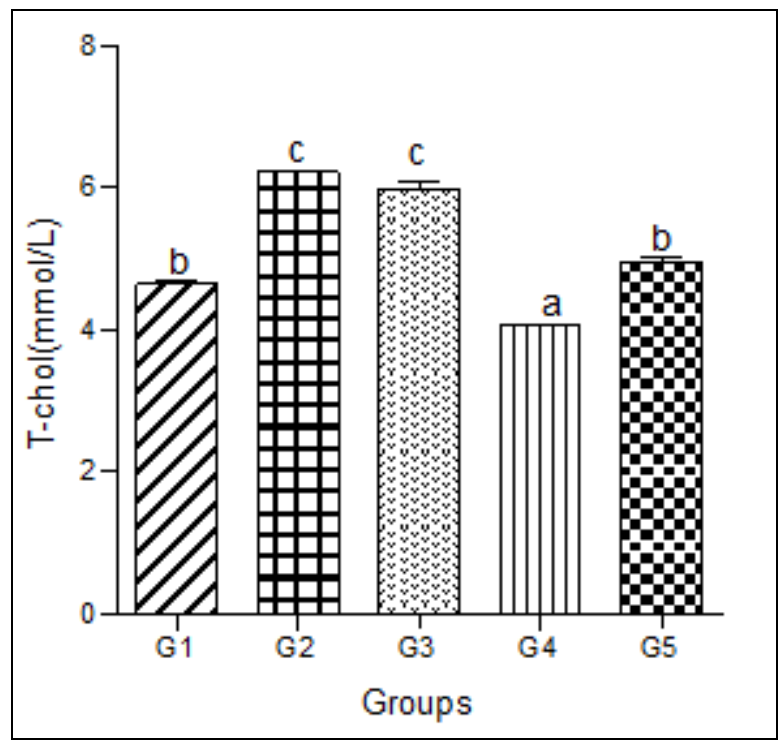

Figure 1: Effect of decaffeinated coffee on serum T-chol in diet-induced dyslipidemia. Values are expressed as Mean \pm SEM at $\mathrm{P}<0.05$ relative to control. Different letters indicate significance while similar letters represent non-significance.

\section{Serum triglyceride-cholesterol (TG-C)}

Serum TG-C significantly increased in the hyperlipidemic diet alone group compared with the control group. Significant increases were also observed in hyperlipidemic diet plus low, medium, and high doses of decaffeinated coffee groups (Figure 2).

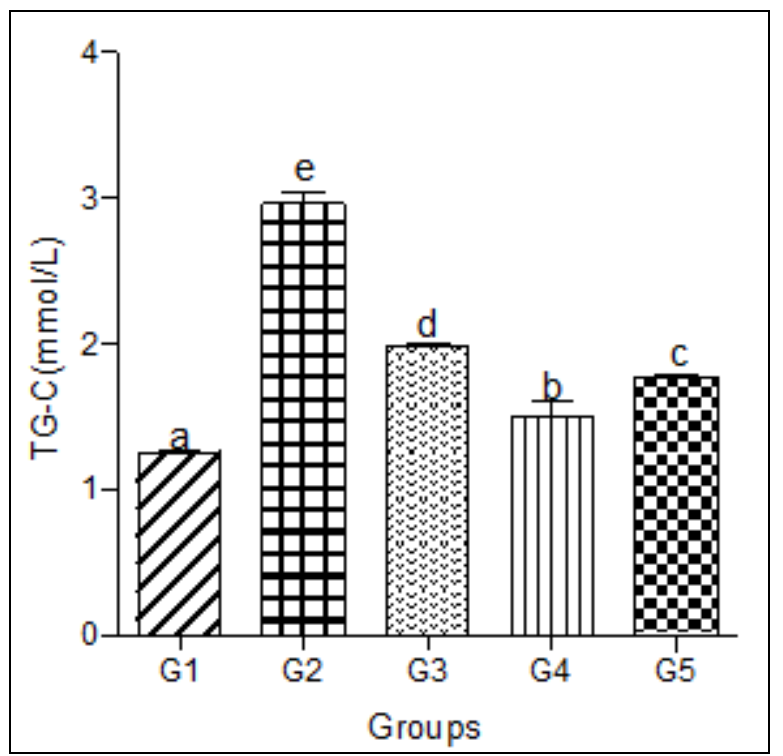

Figure 2: Effect of decaffeinated coffee on serum TG-C in diet-induced dyslipidemia. Values are expressed as Mean \pm
SEM at $\mathrm{P}<0.05$ relative to control. Different letters indicate significance while similar letters represent non-significance.

\section{Serum low-density lipoprotein levels (LDL-C)}

Serum LDL-C increased significantly in the hyperlipidemic diet alone group compared with the control group. Significant increases in serum LDL-C were also observed in the hyperlipidemic diet plus low, medium, and high doses of decaffeinated coffee groups compared with the control group (Figure 3).

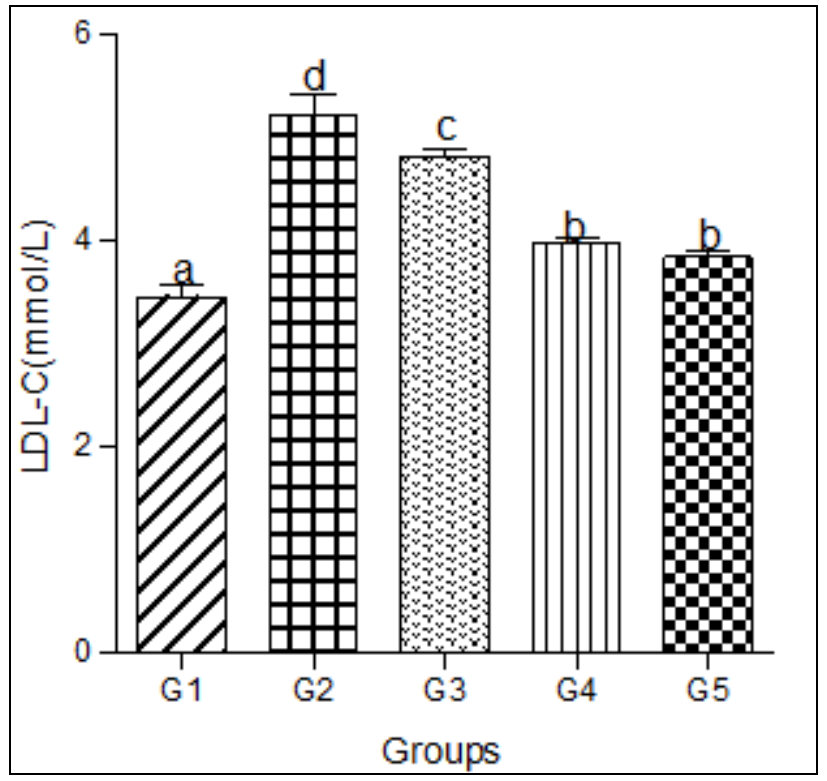

Figure 3: Effect of decaffeinated coffee on serum LDL-C in diet-induced dyslipidemia Values are expressed as Mean \pm $\mathrm{SEM}$ at $\mathrm{P}<0.05$ relative to control. Different letters indicate significance while similar letters represent non-significance.

\section{Serum very-low-density lipoprotein (VLDL-C)}

Animals fed a hyperlipidemic diet alone had a significantly higher level of VLDL-C compared with the control group. When a low dose of decaffeinated coffee was added to the hyperlipidemic diet group, serum VLDL-C was still significantly higher than the control group. However, nonsignificant changes were observed in serum VLDL-C when medium and high doses of decaffeinated coffee were added to the hyperlipidemic diet group compared with the control group (Figure 4). 


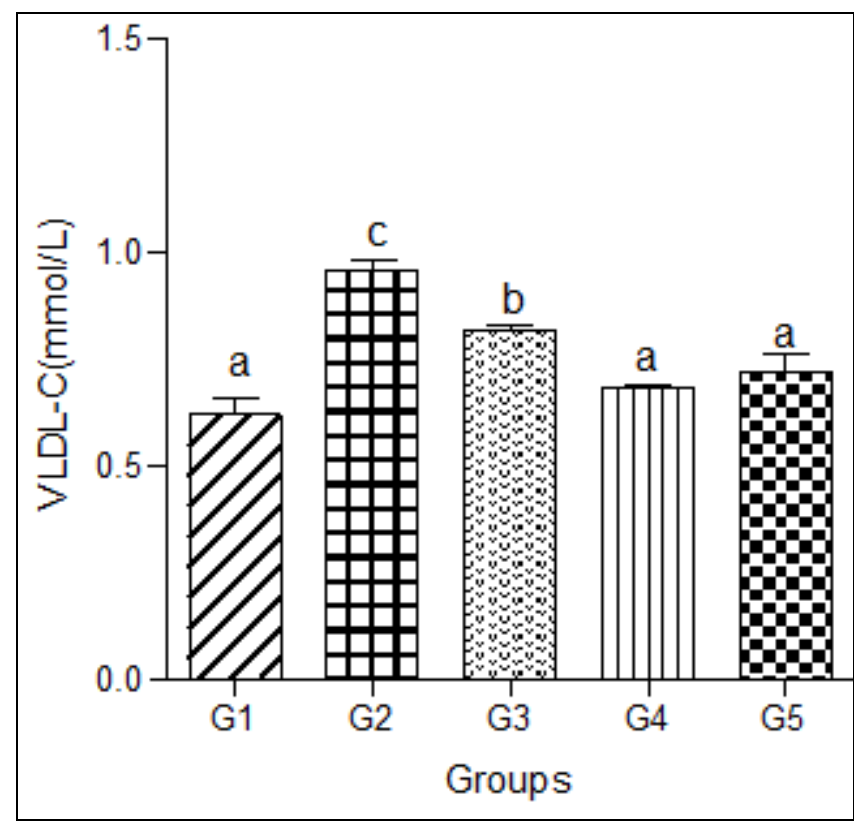

Figure 4: Effect of decaffeinated coffee on serum VLDL-C in diet-induced dyslipidemia. Values are expressed as Mean \pm $\mathrm{SEM}$ at $\mathrm{P}<0.05$ relative to control. Different letters indicate significance while similar letters represent non-significance.

\section{Serum high density lipoprotein-cholesterol (HDL-C)}

Serum HDL-C decreased significantly in the hyperlipidemic diet group compared with the control group. However, when low, medium, and high doses of the decaffeinated coffee were added to the hyperlipidemic diet-fed animals, significant increases were observed compared with the hyperlipidemic diet alone group. These increases were not significantly different when compared with the control group (Figure 5).

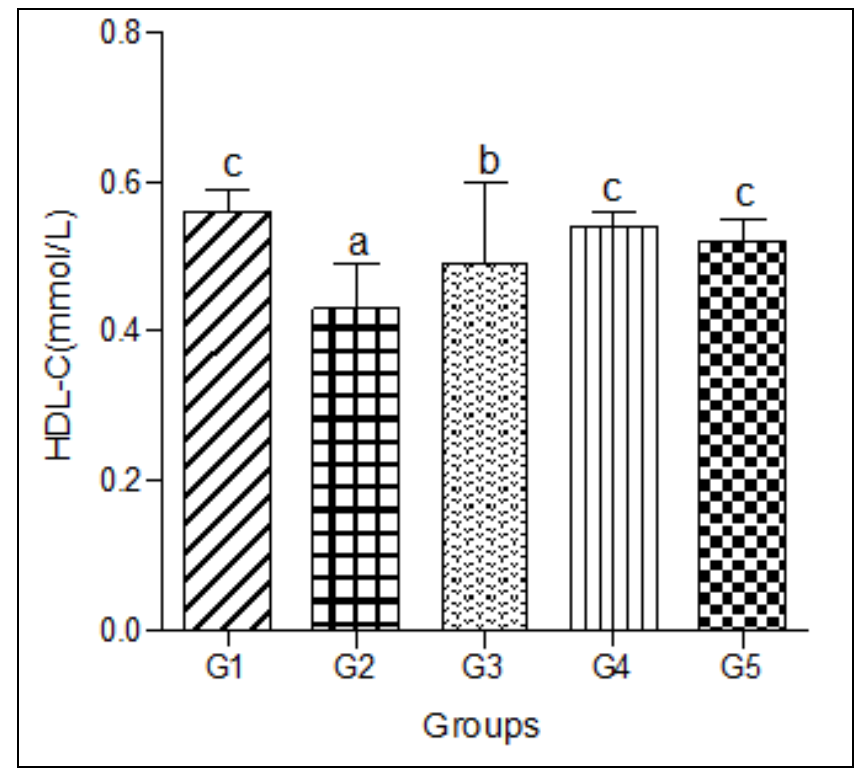

Figure 5: Effect of decaffeinated coffee on serum HDL-C in diet-induced dyslipidemia. Values are expressed as Mean \pm $\mathrm{SEM}$ at $\mathrm{P}<0.05$ relative to control. Different letters indicate significance while similar letters represent non-significance.

\section{Atherogenic Index of Plasma (AIP)}

Animals fed a hyperlipidemic diet showed a significantly higher level of AIP compared with the control group. Coadministration of the low, medium or high doses of decaffeinated coffee caused significant decreases in AIP compared with the hyperlipidemic diet group but were significantly higher than the control group (Figure 6).

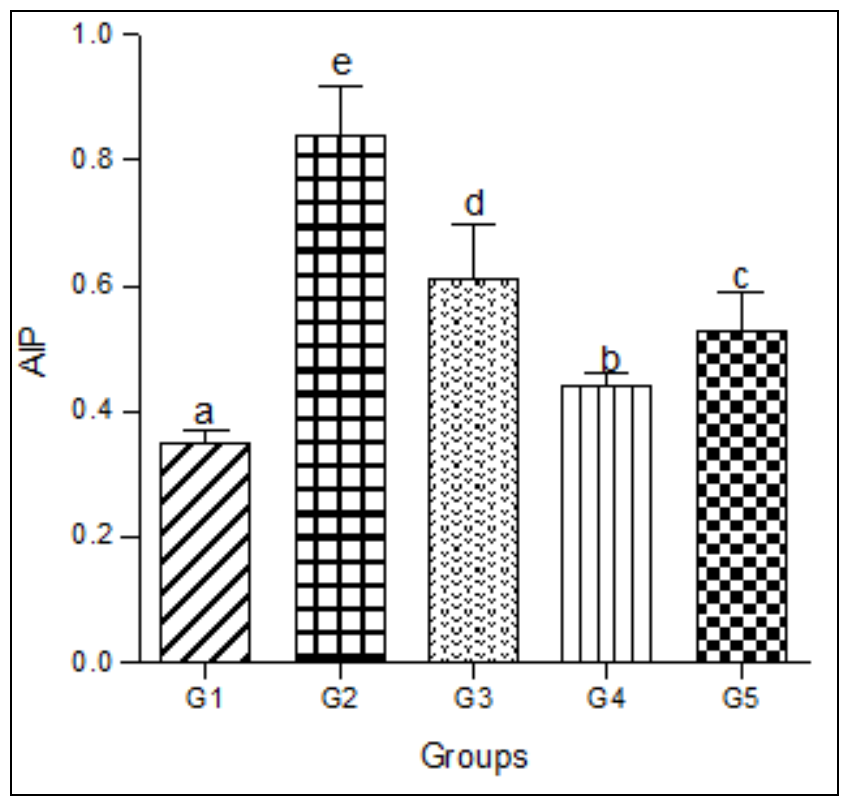

Figure 6: Effect of decaffeinated coffee on AIP in dietinduced dyslipidemia. Values are expressed as Mean \pm SEM

at $\mathrm{P}<0.05$ relative to control. Different letters indicate significance while similar letters represent non-significance.

\section{Serum Uric Acid levels (SUA)}

SUA increased significantly in the animals fed the hyperlipidemic diet alone compared with the control group. Supplementation with low, medium, or high doses of decaffeinated coffee to the hyperlipidemic diet treated groups caused dose-dependent decreases in SUA levels (Figure 7). 


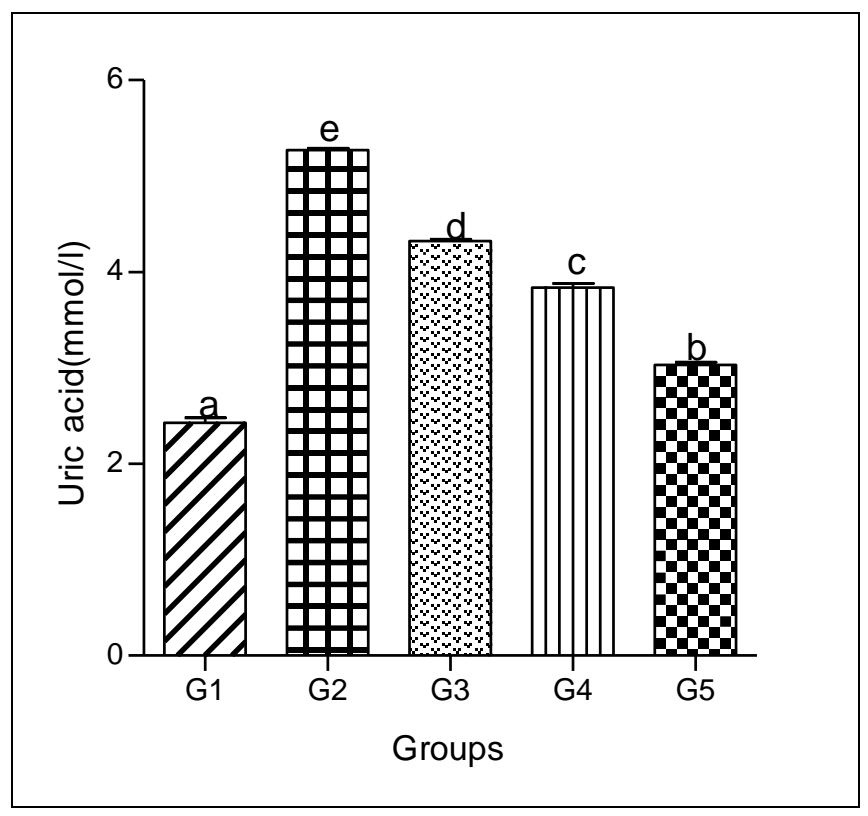

Figure 7: Effect of decaffeinated coffee on SUA in dietinduced dyslipidemia. Values are expressed as Mean \pm SEM at $\mathrm{P}<0.05$ relative to control. Different letters indicate significance while similar letters represent non-significance.

\section{Fasting Blood Glucose levels (FBG)}

Fasting blood glucose levels increased significantly in animals fed a hyperlipidemic diet alone compared with animals in the control group. Animals in the hyperlipidemic diet plus low or high dose of the decaffeinated coffee group showed a significant decrease in FBG level when compared with the hyperlipidemic diet alone group, but a non-significant change in FBG level when compared with the normal control group.

Animals in the hyperlipidemic diet plus medium dose of decaffeinated coffee had a FBG level that did not significantly differ from the hyperlipidemic diet alone group but were significantly higher than the FBG level in the control group (Figure 8).

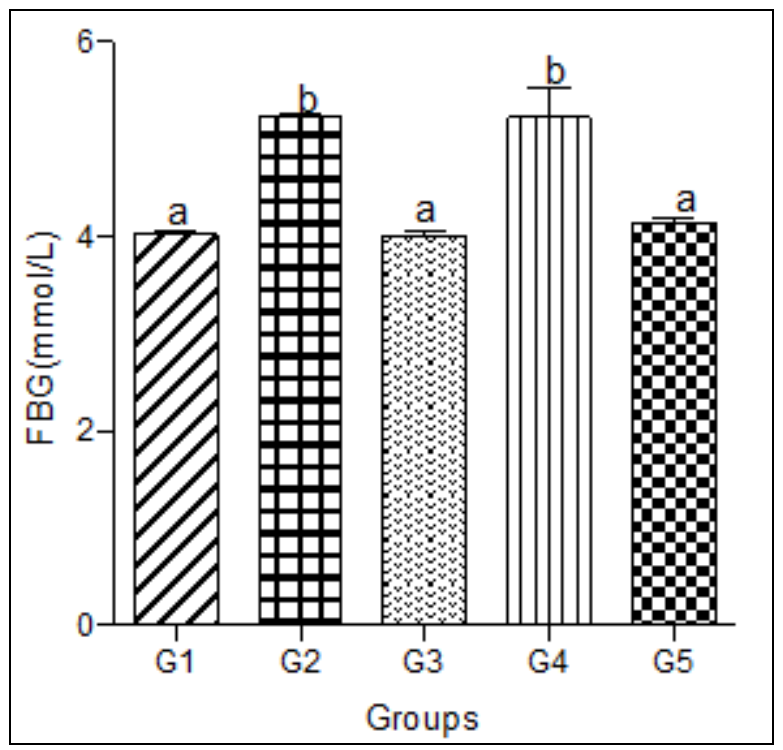

Figure 8: Effect of decaffeinated coffee on FBG in dietinduced dyslipidemia. Values are expressed as Mean \pm SEM at $\mathrm{P}<0.05$ relative to control. Different letters indicate significance while similar letters represent non-significance.

\section{Serum Insulin Concentration}

Fasting insulin level increased significantly in the hyperlipidemic diet group but showed dose-dependent nonsignificant decreases in the low, medium and high decaffeinated coffee treated groups (Figure 9).

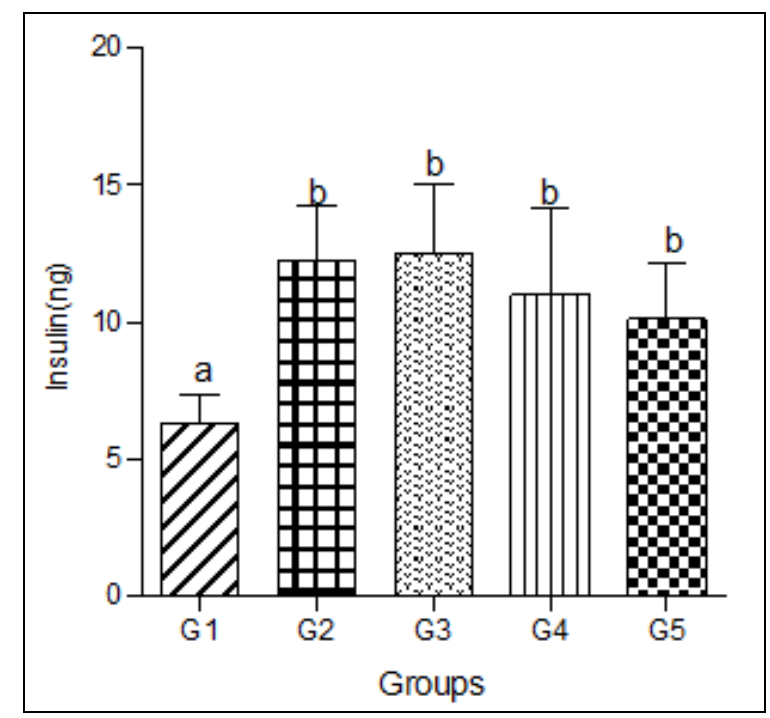

Figure 9: Effect of decaffeinated coffee on serum insulin concentration in diet-induced dyslipidemia. Values are expressed as Mean \pm SEM at $\mathrm{P}<0.05$ relative to control. Different letters indicate significance while similar letters represent non-significance. 


\section{Homeostatic Model Assessment of Insulin Resistance (HOMA- IR)}

HOMA-IR increased significantly $(\mathrm{P}<0.05)$ in animals treated with the hyperlipidemic diet compared with values in the control group. Calculated HOMA-IR dose independently decreased in animals treated with low, medium, and high doses of decaffeinated coffee compared with the hyperlipidemic diet group but significantly increased when compared with the normal group (Figure 10).

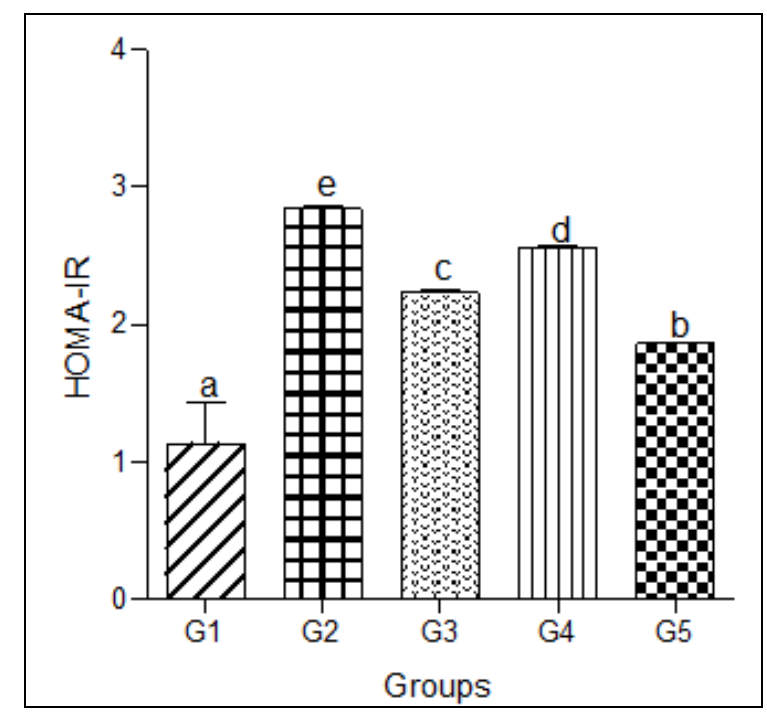

Figure 10: Effect of decaffeinated coffee on HOMA-IR in diet-induced dyslipidemia. Values are expressed as Mean \pm $\mathrm{SEM}$ at $\mathrm{P}<0.05$ relative to control. Different letters indicate significance while similar letters represent non-significance.

\section{Discussion}

The results of the present study revealed that diet plays an important role in the pathogenesis of abnormal serum lipid levels, and hence atherosclerosis-induced cardio-metabolic disorders. Likewise, dietary modification especially, the use of plant-based foods can be applied as an important and early intervention approach to reduce abnormal serum lipid levels, cardiovascular disease risk, and abnormal indices of carbohydrate metabolism (hyperglycemia, and insulin resistance). These notions are supported by the observed abnormalities in mean serum lipid levels (LDL-C, T-chol, TG-C, and a significant decrease in mean serum HDL-C), and markers of carbohydrate metabolism in the hyperlipidemic diet treated group, and the restoration of the serum lipid to near levels in the control group following the administration of decaffeinated coffee. Consistent with these findings, Sadeek et al [8] reported a significant decrease in triacylglycerol, LDL-C, VLDL-C, LDL-C/HDL-C ratio, and TC/HDL-C as well as an increase in serum HDL-C level following the consumption of the coffee drink. Also, Morakinyo and colleagues [9] found that coffee consumption ameliorated high sucrose-induced glucose intolerance, insulin resistance, lipid abnormalities, and oxidative stress in rats.

Given the fact that the vast and versatile pharmacological actions of many medicinal plants are dependent on their bioactive constituents $[\mathbf{1 0}, \mathbf{1 1}]$, we hypothesized that the dyslipidemic modulating effect of the decaffeinated coffee observed in the present study was at least in part mediated by the rich coffee bioactive constituents including vitamins (niacin and vitamin $\mathrm{E}$ ), electrolytes (magnesium $(\mathrm{Mg}$ ) and potassium $(\mathrm{K})$ and phytochemical constituents (tannins and polyphenolic acids (e.g., chlorogenic acid, caffeine, caffeic acid and caffeine metabolites (di-and mono-xanthines, flavonoids melanoidins furans, pyrroles, and maltos) [12]. Correlative studies showed increased serum levels of these compounds following coffee consumption. For instance, Kempt et al., [13] reported an increase in serum levels of chlorogenic acid (CGA), caffeine, caffeic acid metabolites following coffee consumption. Similarly, the consumption of coffee raised the serum level of $\mathrm{Mg}$ in a study carried out by Saito et al., [14]. Some of these compounds are influential antioxidants [9] that attenuate oxidative stress [15], inflammation [16], and immune system dysfunction which are the underlined pathologies driving the onset, progression, and complications of diet-induced abnormalities in serum lipid and carbohydrates metabolism. For instance, CGA, a dietary polyphenol, and a major compound in coffee possess a recognized antioxidant prowess [17]. It reduces superoxide production and directly scavenges free radicals [18]. In one study, intravenous administration of CGAs caused a decrease in serum TG-C levels in Zucker fa/fa rats [19], while the serum level of HDL-C significantly increased. Also, the ratio of LDL-C to HDL-C significantly decreased following the consumption of coffee. Besides its antioxidant effect, CGA is also known to activate the adenosine monophosphateactivated protein kinase (AMPK) signaling pathway which is a key modulator of lipid and glucose metabolism [20]. CGA restored diet-induced risk factors of cardiovascular disease. Similarly, $\mathrm{Mg}$ acts as a cofactor for enzymes associated with lipid metabolisms such as lipoprotein lipase and pyrophosphatase [21]. Deficiency of $\mathrm{Mg}$ is associated with several forms of lipid abnormalities including hypertriglyceridemia, hypercholesterolemia, increased LDL-C, decreased HDL-C, and decreased esterified cholesterol through triglycerides clearance.

The findings of the present study contradict a Turkish study that found no statistically significant alteration in the serum lipid levels (TG-C, LDL-C, HDL-C, OR VLDL-C) amongst 122 Turkish subjects who consumed Turkish coffee and instant coffee even after adjusting for several confounding factors [22]. Also, Zargar et al., [23] found no significant change in serum levels of TC, LDL-C, or non-HDL-C following coffee consumption in an adult population. However, TG-C levels were significantly reduced compared with the normal control group. In other studies $[\mathbf{2 4}, \mathbf{2 5}, \mathbf{2 6}]$ mixed 
changes in serum lipid levels, with no consistent pattern were reported following the consumption of the coffee drink. For instance, in one study, Urgert and Katan [24] found that the consumption of boiled coffee caused a greater dose-dependent increase in TC and LDL-C than filtered coffee, however, the LDL-C level was not affected.

A substantial increase in serum levels of TC and TG-C was reported by Naidoo et al, [26] following the consumption of 5 cups of unfiltered Indonesian coffee per day. In similar manna, the consumption of boiled coffee caused a greater increase in the serum levels of TC and LDL-C, but in a dosedependent manner, while filtered coffee had a relatively lesser effect on the serum level of TG [25].

The observed discrepancies in the effect of coffee on serum lipid levels across studies have been attributed to the effect of coffee bioactive compounds and the varying intake amounts which is determined by several other factors including differences in the method of coffee preparation [27], the quantity of coffee consumed, genetic factors, age, obesity status, sedentary lifestyle, alcohol, smoking habits, gender, and inter-individual differences. These factors have been shown to affect the pharmacokinetic and pharmacodynamic of coffee bioconstituents, hence, the bioavailability and clearance rate. For instance, methods of coffee preparation (e.g., boiling and use of unfiltered coffee) have been shown to affect the concentration of coffee bioactive compounds, and their activities. Boiling and use of unfiltered coffee have been found to increase the serum levels of cholesterol increasing compounds such as diterpenes (cafestol and kahweol) in coffee due to the prolonged contact with hot water, while brewing or filtering causes the depletion of diterpenes by filter paper thereby decreasing serum levels of these substances [28]. This explains why the consumption of boiled or unfiltered coffee was associated with increased serum levels of TC and LDL-C, while brewed/filtered coffee had less effect on serum TC and a null effect on serum LDL-C as reported by Urgert and Katan [24] and Jee et al., [25]. Similarly, instant coffee, but not brewed coffee caused a raised serum LDL-C level and decreased serum TG level [29].

Furthermore, some of the solvents/methods used in the process of decaffeination and the coffee species have been found to cause a decrease in the level of some bioactive compounds and their effects on serum lipid levels. For instance, organic solvent decaffeination was reported to cause CGAs losses of $16 \%$ in Arabica beans and $11 \%$ in Robusta bean [30]. Likewise, CGA losses were reported for the water decaffeination method [31]. Therefore, decaffeination-induced losses of lipid reducing compounds in the face of increasing levels of lipid increasing compounds could produce inconsistent results observed across studies.

According to Naidoo et al [32], the consumption of a large quantity (5cups) of Indonesia coffee/day caused a substantial increase in serum levels of TC and TG, and hence increased risk of coronary heart disease (CHD). Whereas, moderate coffee consumption ( $>1$ or 2 cups daily) significantly lowered the risk of CHD in both men and women [32]. Accordingly, studies conducted in Germany and Israel observed a dosedependent increase in TC in a population of young people [33, 34]. Therefore, the absence of effect of coffee on serum lipid levels could be a dose-dependent effect as also posited by Karabudak et al., [22]. Coffee consumption habits (e.g., coffee consumption plus smoking) and differences in genotypes and genetic environment have been shown to affect the effect of coffee on serum lipid levels $[\mathbf{2 2}, \mathbf{3 5}]$. The caffeine content of the experimental coffee sample has been posited to affect the changes in serum lipid profile. One study showed that the consumption of $750-1000 \mathrm{ml}$ of decaffeinated coffee failed to cause any significant alteration in serum levels of TG, TC, LDL-C, and Apo B when compared with caffeinated coffee [36], further supporting the results of the present study.

However, inconsistent results have been obtained from studies examining the effect of coffee consumption habits or the addition of flavoring substances on the serum lipid levels. For instance, while Karabudak et al., [22] failed to observe the effect of adding sugarcane and liquid/power cream to coffee on serum lipid levels, Cheung et al., [37] found that the addition of flavoring substances (sugar and cream) to coffee caused significant changes in the serum levels of TC, HDL-C, and TG, but not serum level of LDL-C.

Similarly, while the consumption of coffee alongside cigarette smoking failed to cause changes in serum lipid levels in some studies, Salonen et al., [38], reported that high consumption of coffee caused a decrease and increase in serum HDL-C among smokers, but not in non-smokers respectively.

We also observed that although serum lipid levels improved following coffee consumption, the levels were not as good as those of the control group, supporting the result of a meta-analysis of 14 randomized clinical trials that showed that patients with hyperlipidemia tend to have higher lipid levels after coffee consumption than healthy subjects [25].

\section{Conclusion}

Moderate consumption of decaffeinated coffee could ameliorate diet-induced abnormalities in lipid and carbohydrate metabolism corollary of its bioactive constituents and their activities.

Authors' Contributions: CEE conducted the experimental part and elaborated the script; DEO reviewed the literature and analyzed data.

Funding: This research received no external funding.

Conflicts of Interest: The authors declare no conflicts of interest. 


\section{References}

1. Rosamond W, Flegal K, Furie K, Go A, Greenlund K et al. (2008) Heart disease and stroke statistics--2008 update: a report from the American Heart Association Statistics Committee and Stroke Statistics Subcommittee. Circulation 117:25-146.

2. Ivanova EA, Myasoedova VA, Melnichenko AA, Grechko AV, Orekhov AN (2017) Small Dense LowDensity Lipoprotein as Biomarker for Atherosclerotic Diseases. Oxf Cell Longev 1273042.

3. Ekpenyong CE, Patrick ES, Akpan KS (2017) Changes in serum markers of atherogenesis and hematological profile after the consumption of quail eggs. Archive of Food and Nutritional Sciences 1:001-011.

4. Leitzman MF, Willett WC, Rimm EB, Stampfer MJ, Spiegelman D et al. (1999) A Prospective Study of Coffee Consumption and the Risk of Symptomatic Gallstone Disease in Men. JAMA 281:2106-2112.

5. Ekpenyong CE, Davies K, Antai EE (2014) Cymbopogon citratus Stapf (DC) Extract Ameliorates Atherogenic Cardiovascular Risk in Diabetes-Induced Dyslipidemia in Rats. British Journal of Medicine and Medical Research 4:4695-4709.

6. Gandhi VM, Cherian KM, Mulky MJ (1992) Hypolipidemic action of taurine in rats. Ind J Exp Bio 413-417.

7. Friedewald WT, Levy RI, Fredrickson DS (1972) Estimation of the concentration of low-density lipoprotein cholesterol in plasma, without use of the preparative ultracentrifuge. Clin Chem 18:499-502.

8. Sadeek EA, El-Rahman HAA, Ali WS (2010) The Effect of Green, Roasted and Decaffeinated Coffee on Serum Glucose, Insulin and Serum Lipid Profile in Diabetic Experimental Animals. Journal of American Science 6: 934-943.

9. Morakinyo AO, Adekunbi DA, Dada KA, Adegoke OA (2013) Coffee Consumption Attenuates Insulin Resistance and Glucose Intolerance in Rats fed on High-Sucrose Diet. Niger J Physiol Sci 28:179-185.

10. Ekpenyong CE, Essiet U (2019) Cymbopogon citratus Stapf (DC) extract attenuates gasoline vapourinduced low-triiodothyronine syndrome, oxidative stress and lipid peroxidation in rats. Trends in Phytochemistry research 3:189-200.

11. Hussein RA, El-Anssary AA (2018) Plants secondary metabolites: The key drivers of the pharmacological actions of medicinal plants. Intech Open.

12. Ekpenyong CE, Nwachuku C, Otakpa DE (2020) Decaffeinated Coffee Consumption and Renal Function Impairment in Health and Diabetes: A Paradigm-Shift. Advancement in Journal of Urology and Nephrology 2: 78-87.

13. Kempf K, Herder C, Erlund I, Kolb H, Martin S et al (2010) Effects of coffee consumption on subclinical inflammation and other risk factors for type 2 diabetes: a clinical trial. Am J Clin Nutr 91: 950-957.

14. Saito $M$, Nemoto $T$, Tobimatsu $S$, Ebata $M$, Le $Y$ et al. (2011) Coffee Consumption and Cystatin-C-Based Estimated Glomerular Filtration Rates in Healthy Young Adults: Results of a Clinical Trial. Journal of Nutrition and Metabolism 146865.

15. Gomez-Ruiz JA, Leake DS, Ames JM (2007) Invitro antioxidant activity of coffee compounds and their metabolites. J Agric Food Chem 55:6962-6969.

16. Lopez-Garcia E, Van Dam RM, Qi L, Hu FB (2006) Coffee consumption and markers of inflammation and endothelial dysfunction in healthy and diabetic women. AM J Clin Nutr 84: 888-893.

17. Tajik N, Tajik M, Mack I, Enok P (2017) The potential effects of chlorogenic acid, the main phenolic components in coffee, on health: a comprehensive review of the literature. Eur J Nutr 56:2215-2244.

18. Lim D, Chang J, Ahn J, Kim J (2020) Conflicting effects of coffee consumption on cardiovascular diseases: Does coffee consumption aggravate pre-existing risk factors? Processes 8:438.

19. Shimoda H, Seki E, Aitani M (2006) Inhibitory effect of green coffee bean extract on fat accumulation and body weight gain in mice. BMC Complementary and Alternative Medicine 6:9.

20. Meng S, Cao J, Feng Q, Peng J, Hu Y (2013) Roles of chlorogenic acid on regulating glucose and lipids metabolism: A review. Evid-based complementary and alternative Medicine 801457.

21. Deepti R, Nalini G, Anbazhagan (2014) Relationship between Hypomagnesemia and Dyslipidemia in Type 2 Diabetes Mellitus. Asian J Pharmaceutical Research and Health Care 6:32-36.

22. Karabudak E, Turkozu D, Koksal E (2015) Association between coffee consumption and serum lipid profile. Experimental and Therapeutic Medicine 9:1841-1846.

23. Zargar A, Auttapibarn C, Hong SH, Larson TJ, Hayworth $\mathrm{KN}$ et al. (2013) The effect of acute caffee latte ingestion on fasting serum lipid levels in healthy individuals. J Clin Lipidol 7:165-168.

24. Urgert R, Katan MB (1996) The cholesterol-raising factor from coffee beans. J R Soc Med 89:618-623.

25. Jee SH, He J, Appel LJ, Wheiton PK, Suh I et al. (2001) Coffee Consumption and Serum Lipids: A Meta-Analysis of Randomized Controlled Clinical Trials. Am J Epidemiol 153:353-362.

26. Naidoo N, Chen C, Rebello SA, Speer K, Tai ES et al. (2011) cholesterol-raising diterpenes in types of coffee commonly consumed in Singapore, Indonesia, and India and associations with blood lipids: A survey and crosssectional study. Nutr J 10:48.

27. Correa TA, Rogero MM, Mioto BM, Tarasoutchi D, Tuda VL et al. (2013) Paper-filtered coffee increases cholesterol and inflammation biomarkers independent of roasting degree: a clinical trial. Nutrition 29:977-981. 
28. Lombardi M, Cruciani G, Mazza A, Luzi L, Leggio M (2014) Coffee consumption and cardiovascular risk: an updated overview. J Cardiology Therapy 1:200-205.

29. Miyake $\mathrm{Y}$, Kono S, Nishiwaki M, Hamada H, Nishikawa $\mathrm{H}$ et al. (1999) Relationship of coffee consumption with serum lipids and lipoproteins in Japanese men. Annals of Epidemiol 9:121-126.

30. Toci A, Farah A, Trugo LC (2006) Effect of decaffeination using dichloromethane on the chemical composition of Arabica and robusta raw and roasted coffees. Quim Nova SBQ 29:965-971.

31. Farab A (2012) Coffee constituents. In: Chu Y-F, editor. Coffee: emerging health effects and disease prevention. Oxford, UK, Wiley-Black well.

32. Wu JN, Ho SC, Zhou C, Ling WH, Chem WQ et al. (2009) Coffee consumption and risk of coronary heart diseases: a meta-analysis of 21 prospective cohort studies. Int J Cardiol 137:216-225.

33. Green MS, Jucha E (1986) Association of serum lipids with coffee, tea, and egg consumption in free-living subjects. J Epidemiol Community Health 40:324-329.
34. Kohlmeier L, Mensink G, Kohlmeier M (1991) The relationship between coffee consumption and lipid levels in young and older people in the Heidelberg-MichelstadtBerlin study. Eur Heart J 12:869-874.

35. Nilsson LM, Wennberg $\mathrm{M}$, Lindahl $\mathrm{B}$, Eliasson $\mathrm{M}$, Jansson JH et al. (2010) Consumption of filtered and boiled coffee and the risk of first acute myocardial infarction; a nested case/referent study. Nutr Metab Cardiovascular Dis 20:527-535.

36. Boekschoten MV, Engherink MF, Katan MB, Schouten EG (2003) Reproducibility of the serum lipid response to coffee oil in healthy volunteers. Nutr J 2:8.

37. Cheung RJ, Gupta EK, Ito MK (2005) Acute coffee ingestion does not affect LDL cholesterol level. Ann Pharmacother 39:1209-1213.

38. Salonen JT, Happonen P, Salonen R, Korhonen H, Nissinen A et al. (1987) Interdependence of associations of physical activity, smoking, and alcohol and coffee consumption with serum high-density lipoprotein and non-high-density lipoprotein cholesterol--a population study in eastern Finland. Pre Med 16:647-658.

Citation: Ekpenyong CE, Otokpa DE (2020) Effect of Decaffeinated Coffee Consumption on Markers of Atherogenesis and Carbohydrate Metabolism in Diet-Induced Dyslipidemia in Rats. Adv in Nutri and Food Sci: ANAFS-194. 